\title{
Triply Differential Ionization Cross Sections of atomic and molecular targets by single electron impact
}

\author{
Lena Mouawad*, Ziad El Bitar \\ Université de Strasbourg, CNRS \\ IPHC UMR 7178, F-67000 \\ Strasbourg, France \\ Email*: lena.mouawad@iphc.cnrs.fr
}

Paul Antoine Hervieux

Institut de Physique et Chimie des Matériaux de Strasbourg

23 rue du Loess, BP 43, 67034

Strasbourg, France

\author{
Lena Mouawad, Ahmad Osman, Mohamad Khalil \\ AZM research center, EDST \\ Lebanese University \\ Tripoli, Lebanon
}

\author{
Claude Dal Cappello \\ Laboratoire de Physique Moléculaire et des Collisions \\ UMR CNRS 7565, Université de Lorraine \\ 1 Boulevard Arago, 57078 Metz Cedex 3, France
}

\begin{abstract}
We present numerically calculated triply differential cross sections for the simple ionization of two targets, atomic hydrogen and ammonia, in the First Born Approximation framework with one Coulomb wave describing the ejected electron. The partial wave series of the wavefunctions centered around the same origin were used and the intial state molecular wavefunction was derived using information provided by Gaussian03 software for ammonia. The theoretical results are calculated in the geometrical settings and kinematical conditions of previous experiments and are compared to published experimental and theoretical data. The results show good agreement with previous studies based on the same model for hydrogen. The general shape of the triply differential cross sections obtained for ammonia are in acceptable agreement with experimental data in the binary region but not in the recoil region where our simple framework fails to reconstruct the recoil peaks.
\end{abstract}

\section{INTRODUCTION}

The study of the ionization of atoms and molecules by electron impact is necessary to reveal information about the structure of the target and improve the understanding of particle interactions in many domains. The dynamics of this process is best described by the triply differential cross section (TDCS) which involves all the kinematic parameters of ionization providing the ionization probability over the ejected electron angle distribution. Determining this quantity presents many challenges both experimentally and theoretically because it requires an accurate description of the evolution of all the electrons' states that are involved as well as their mutual interactions before and after collision. Modeling these interactions becomes even more complex in the case of polyatomic molecular targets.

Extensive research has been done to provide TDCSs for a variety of atomic and molecular targets exploring different theoretical models. At high incident and ejected electron energies, the nucleus's role can be neglected and the interaction can be approximated by a simple Coulomb attraction between the ejected electron and the nucleus. Under these kinematics, the use of the first Born approximation (FBA) is justified and slight improvements are provided by other much more sophisticated models that require heavy calculations. To further simplify the calculations, many groups have used partial wave expansion which facilitates the translation of the analytical calculations to parallel numerical codes with the least computing time possible [1]-[3].

Accurately describing the molecular wave function is very important and presents many challenges for multicenter complex molecules. Defining the wave functions around a single center reduces the complexity of this task [4]. These wave functions have been expressed as linear combinations of atomic orbitals written in terms of Slater-type functions in some studies [5], [6] and in terms of Gaussian-type functions in others [3], [7]. Although Slater-type functions provide a more accurate desription of the atomic orbitals, they are difficult to express on distinct atoms of bigger molecules and hence the use of Gaussian-type orbitals is preferrable to describe molecular wave functions. Another challenge that is faced when dealing with molecular targets is defining the molecule's orientation before the collision. This cannot be done in experiments, therefore an average orientation of the molecule should be considered in theoretical calculations for appropriate comparision with experimental data. This can be done either by using orientation averaged molecular orbitals (OAMO) [8] or by calculating TDCSs for each possible orientation and then determining the average TDCS [9]. In this work, we use single center Gaussian-type molecular wave functions developed from the GAUSSIAN 03 [10] program in the 6$31 \mathrm{G}$ basis set. 
The hydrogen atom represents the simplest case study that allows the validation of developed analytical and numerical models before moving on to molecular targets which intial state wave functions are more complex to determine. Ehrhardt et al. performed an experiment in which they measured the TDCSs of atomic hydrogen at incident energy of $250 \mathrm{eV}$, ejected energy of $5 \mathrm{eV}$ and a detection angle of the scattered electron equal to 3 degrees and 8 degrees [11]. The experimental data showed a binary peak near the direct momentum transfer direction and a recoil peak near its backward direction. In 2012 Sahlaoui and Bouamoud [6] compared analytical TDCSs calculated within the FBA framework using Slatertype functions to these experimental data. Their work aimed at proposing an improvement of the FBA to model postcollision interactions by multiplying the TDCS equation with the Gamow factor [12]. We consider the results of these studies and compare them to our TDCSs for the ionization of the hydrogen atom. We also present calculated TDCSs for the ionization of ammonia and compare them to the experimental data of El Mir et al. [13] measured at an incident energy of $500 \mathrm{eV}$, ejected energy of $74 \mathrm{eV}$ and with scattered angle of -6 degrees. These data show the same trend observed for the hydrogen atom TDCSs. In the work of El Mir et al. these data were compared to analytical cross sections calculated within the FBA using the one coulomb wave model for the ejected electron with Slater-type wave functions developed according to the approach proposed by Moccia [14]. They also presented theoretical results using a distorted wave to describe the ejected electron and results calculated with the Brauner-Briggs-Klar model (BBK) [15]. The FBA results also underestimated the recoil peak but predicted a better binary to recoil amplitude ratio than the other models for the $3 \mathrm{a}_{1}$ and $1 \mathrm{e}_{1}$ orbitals. We present numerical TDCSs calculated also in the FBA-1CW framework but with Gaussian-type wave functions and writing all the wave functions in partial wave series and compare our results to the experimental and analytical data of El Mir et al. [13].

We first begin by presenting our theoretical framework, then we provide our results for both atomic hydrogen and ammonia. Our results show very good agreement with published analytical data therebye validating our program.

\section{THEORETICAL FRAMEWORK}

The triply differential cross section $\sigma^{(3)}$ is given by:

$$
\sigma^{(3)}=\frac{k_{f} k_{e}}{k_{i}}\left|f_{B 1}\right|^{2}
$$

$f_{B 1}$ being the transition amplitude in the first born approximation from the state $\Psi_{i}$ to the state $\Psi_{f}$ :

$$
f_{B 1}=\frac{-1}{2 \pi}<\Psi_{f}|V| \Psi_{i}>
$$

The interaction potential $V$ is the coulomb interaction between the incident electron and the target noting $\vec{R}$ as the position vector of the heaviest nucleus of the target with respect to the incident electron. In the case of the hydrogen atom, the interaction potential is simply written as:

$$
V=-\frac{1}{\vec{R}}+\frac{1}{\left|\vec{R}-\overrightarrow{r_{1}}\right|}
$$

$\overrightarrow{r_{1}}$ being the position vector of the bound electron $e_{1}$ with respect to the Hydrogen atom.

On the other hand, the potential interaction between the incident electron and the $\mathrm{NH}_{3}$ molecule is given by:

$$
\begin{gathered}
V=-\frac{7}{\vec{R}}-\frac{1}{\left|\vec{R}-\overrightarrow{R_{1}}\right|}-\frac{1}{\left|\vec{R}-\overrightarrow{R_{2}}\right|}-\frac{1}{\left|\vec{R}-\overrightarrow{R_{3}}\right|} \\
+\sum_{i=1}^{10} \frac{1}{\left|\vec{R}-\overrightarrow{r_{i}}\right|}
\end{gathered}
$$

$\vec{R}$ is the position vector of the Nitrogen atom with respect to the incident electron, $\overrightarrow{R_{1}}, \overrightarrow{R_{2}}$ and $\overrightarrow{R_{3}}$ are the position vectors of the three Hydrogen atoms with respect to the Nitrogen atom, and $\overrightarrow{r_{i}}$ is the position vector of the ith bound electron with respect to the Nitrogen atom.

In the First Born Approximation the incident and scattered electrons are both described by plane waves, $e^{i \overrightarrow{K_{i}} \vec{R}}$ and $e^{i \overrightarrow{K_{f}} \vec{R}}$ respectively, while the ejected electron is described by a Coulomb wave $\mathscr{F}_{K_{\boldsymbol{e}}}^{(-)}(\vec{r})$.

Let's consider the molecular target case of ammonia. Before collision, the target electron is bound to a molecular orbital $i$ and is described by the molecular orbital wavefunction $\boldsymbol{\psi}_{\boldsymbol{i}}(\boldsymbol{r})$. The method used to develop the molecular orbital wavefunctions from Gaussian03 was presented in details elsewhere [16], [17] so it will not be discussed here. In summary, the contracted Cartesian Gaussian type orbitals (CGTO), used as basis functions in Gaussian, are written as linear combinations of primitive gaussian type orbitals which are then written as function of spherical type orbitals. The obtained form for the wavefunction is:

$$
\psi_{i}(x, y, z)=\sum_{\kappa=1}^{n_{c}} \sum_{j=1}^{n_{p}} \sum_{n, l, m} a_{i k} d_{\kappa j} A(i j k, n l m) \phi_{b}(\hat{r})
$$

A single center partial wave expansion of the molecular wavefunction is then performed thereby reducing the complexity of studying a multicenter molecule and that by separating the radial and angular components of the spherical gaussian functions. This was already done in details by Kaufmann and Baumeister [4] where a detailed form of the spherical gaussian 
functions is given. This leads to a single-center molecular orbital wavefunction of the following form:

$$
\psi_{i}(x, y, z)=\sum_{\lambda, m_{\lambda}} \tilde{R}_{\lambda, m_{\lambda}}^{i}(r) Y_{\lambda, m_{\lambda}}\left(\Omega_{\vec{r}}\right)
$$

\section{$\vec{r}$ being the position vector with respect to the origin.}

For appropriate comparision with experimental data, the molecular orientation considered in Gaussian is transformed to the laboratry frame by using the rotation operator $\mathscr{R}_{\mu m_{\lambda}}^{\lambda}(\alpha \beta \gamma)$ according to the following:

$$
\psi_{i}(x, y, z)=\sum_{\lambda, m_{\lambda}} \tilde{R}_{\lambda, m_{\lambda}}^{i}(r) \sum_{\mu=-\lambda}^{\mu=+\lambda} \mathscr{R}_{\mu m_{\lambda}}^{\lambda}(\alpha \beta \gamma) Y_{\lambda \mu}(\hat{r})
$$

The initial state wavefunction is the product of the incident electron's plane wave $e^{i \overrightarrow{k_{i}} \vec{R}}$ with the intial state molecular wavefunction $\psi_{\text {is }}$ describing the 10 bound electrons of the ammonia molecule:

$$
\Psi_{i}=e^{i \overrightarrow{k_{i}} \vec{R}} \psi_{i s}\left(\overrightarrow{r_{1}}, \overrightarrow{r_{2}}, \overrightarrow{r_{3}}, . ., \overrightarrow{r_{10}}\right)
$$

With $\psi_{i s}\left(\overrightarrow{r_{1}}, \overrightarrow{r_{2}}, \overrightarrow{r_{3}}, . ., \overrightarrow{r_{10}}\right)=\psi\left(\overrightarrow{r_{1}}\right) \psi\left(\overrightarrow{r_{2}}\right) \psi\left(\overrightarrow{r_{3}}\right) \ldots \psi\left(\overrightarrow{r_{10}}\right)$

After collision, the final state wavefunction is the product of the scattered electron's plane wavefunction $e^{i \overrightarrow{k_{f}} \vec{R}}$ with the ejected electron's Coulomb wavefunction $\mathscr{F}_{k_{e}}^{(-)}\left(\overrightarrow{r_{1}}\right)$ and the molecular wavefunctions of the other 9 bound electrons. We suppose that the electron $e_{1}$ is the ejected electron, with $\overrightarrow{r_{1}}$ being the position vector of $e_{1}$ with respect to the Nitrogen nucleus, and that all other electrons are passive electrons with frozen wavefunctions from the initial to the final state:

$$
\Psi_{f}=e^{i \overrightarrow{k_{f}} \vec{R}} \psi_{f s}\left(\overrightarrow{r_{1}}, \overrightarrow{r_{2}}, \overrightarrow{r_{3}}, . ., \overrightarrow{r_{10}}\right)
$$

With $\psi_{f s}\left(\overrightarrow{r_{1}}, \overrightarrow{r_{2}}, . ., \overrightarrow{r_{10}}\right)=\mathscr{F}_{k_{e}}^{(-)}\left(\overrightarrow{r_{1}}\right) \psi\left(\overrightarrow{r_{2}}\right) \psi\left(\overrightarrow{r_{3}}\right) \ldots \psi\left(\overrightarrow{r_{10}}\right)$

After replacing 4, 8 and 9 in equation 2, and assuming a small momentum transfer from the incident electron to the target, the transition amplitude expression simplifies to that obtained with the simple potential of the Hydrogen atom:

$$
\begin{gathered}
f_{B 1}=\frac{-2}{q^{2}}<\mathscr{F}_{k_{e}}^{(-)}\left(\overrightarrow{r_{1}}\right)\left|e^{i \vec{q} \overrightarrow{r_{1}}}-1\right| \psi\left(\overrightarrow{r_{1}}\right)> \\
\vec{q}=\overrightarrow{k_{i}}-\overrightarrow{k_{f}} \text { is the transfer momentum from the incident } \\
\text { electron to the target. }
\end{gathered}
$$

The plane, Coulomb and target's wavefunctions are written in partial wave expansion as follows:

$$
\begin{gathered}
\mathscr{F}_{\overrightarrow{k_{e}}}^{(-) *}\left(\overrightarrow{r_{1}}\right)=\frac{1}{(2 \pi)^{\frac{3}{2}}} \sum_{l_{e}, m_{e}}(4 \pi)(-i)^{l_{e}} e^{i \delta_{l_{e}}} \frac{F_{l_{e}}\left(k_{e} r_{1}\right)}{k_{e} r_{1}} \\
Y_{l_{e}, m_{e}}\left(\hat{k_{e}}\right) Y_{l_{e}, m_{e}}^{*}\left(\hat{r_{1}}\right) \\
e^{i \vec{q} \vec{r}}=\sum_{l, m}(4 \pi) i^{l} j_{l}(q r) Y_{l, m}^{*}(\hat{q}) Y_{l, m}(\hat{r})
\end{gathered}
$$

$\psi\left(\overrightarrow{r_{1}}\right)=\sum_{\lambda, m_{\lambda}} \tilde{R}_{\lambda, m_{\lambda}}^{i}\left(r_{1}\right) \sum_{\mu=-\lambda}^{\mu=+\lambda} \mathscr{R}_{\mu m_{\lambda}}^{\lambda}(\alpha \beta \gamma) Y_{\lambda \mu}\left(\hat{r_{1}}\right)$

After replacing in 1 , the triply differential cross section becomes:

$$
\begin{array}{r}
\sigma^{(3)}=\frac{k_{f} k_{e}}{k_{i}} \frac{2^{5}}{q^{4}} \mid \sum_{\lambda, m_{\lambda}} \sum_{\mu} \sum_{l, m} \sum_{l_{e}, m_{e}}(-1)^{m_{e}} i^{l-l_{e}} e^{i \delta_{l_{e}}} Y_{l, m}^{*}(\hat{K}) \\
Y_{l_{e}, m_{e}}\left(\left.\hat{\left.k_{e}\right)} \mathscr{R}_{\mu m_{\lambda}}^{\lambda}(\alpha \beta \gamma) \mathcal{R}_{l e, l, \lambda, m_{\lambda}}^{i} \mathcal{A}_{l_{e}, m_{e}, l}^{\lambda, \mu}\right|^{2}\right. \\
(14)
\end{array}
$$

With $\mathcal{R}_{l e, l, \lambda, m_{\lambda}}^{i}=\int r_{1}^{2} \mathrm{~d} r_{1} \tilde{R}_{\lambda m \lambda}^{i}\left(r_{1}\right) \frac{F_{l_{e}}\left(k_{e} r_{1}\right)}{k_{e} r_{1}}\left(j_{l}(q r)-\delta_{l, 0}\right)$ and $\mathcal{A}_{l_{e}, m_{e}, l}^{\lambda, \mu}=\left(\hat{l_{e}} \hat{l} \hat{\lambda}\right)^{\frac{1}{2}}\left(\begin{array}{ccc}l_{e} & l & \lambda \\ 0 & 0 & 0\end{array}\right)\left(\begin{array}{ccc}l_{e} & l & \lambda \\ -m_{e} & m_{e}-\mu & \mu\end{array}\right)$

The rotation operator $\mathscr{R}_{\mu m_{\lambda}}^{\lambda}(\alpha \beta \gamma)$ is represented by a rotation matrix $D_{\mu m_{\lambda}}^{\lambda}(\alpha \beta \gamma)$ such that:

$$
\mathscr{R}_{\mu m_{\lambda}}^{\lambda}(\alpha \beta \gamma)=e^{-i \alpha m} D_{\mu m_{\lambda}}^{\lambda}(\alpha \beta \gamma) e^{-i \gamma m u}
$$

The elements of the rotation matrix $D_{\mu m_{\lambda}}^{\lambda}(\alpha \beta \gamma)$ form a complete set of orthogonal functions of the Euler angles:

$$
\begin{gathered}
\int_{0}^{2 \pi} \mathrm{d} \alpha \int_{0}^{\pi} \sin (\beta) \mathrm{d} \beta \int_{0}^{2 \pi} D_{\mu m_{\lambda}}^{\lambda}(\alpha \beta \gamma) D_{\mu^{\prime} m_{\lambda^{\prime}}^{\prime}}^{\lambda^{\prime *}}(\alpha \beta \gamma) \mathrm{d} \gamma \\
=8 \pi^{2} \frac{1}{\hat{\lambda}} \delta_{\lambda \lambda^{\prime}} \delta_{\mu \mu^{\prime}} \delta_{m_{\lambda} m_{\lambda^{\prime}}^{\prime}}
\end{gathered}
$$

$$
\delta_{\lambda \lambda^{\prime}}, \delta_{\mu \mu^{\prime}}, \delta_{m_{\lambda} m_{\lambda^{\prime}}^{\prime}} \neq 0 \text { for } \lambda=\lambda^{\prime}, \mu=\mu^{\prime}, m_{\lambda}=m_{\lambda^{\prime}}^{\prime}
$$

Integrating 14 using 15 and 16 leads to the following final expression for the TDCS:

$$
\sigma^{(3)}=\frac{k_{f} k_{e}}{k_{i}} \frac{2^{5}}{q^{4}} \sum_{\lambda, m_{\lambda}} \sum_{\mu} \frac{1}{\hat{\lambda}}\left|\mathcal{S}_{\lambda, m_{\lambda}, \mu}^{i}\right|^{2}
$$

With $\mathcal{S}_{\lambda, m_{\lambda}, \mu}^{i}=\sum_{l_{e}, m_{e}} \sum_{l}(-1)^{\mu} i^{\left(l-l_{e}\right)} e^{i \delta_{l_{e}}} Y_{l_{e}, m_{e}}\left(\hat{k_{e}}\right)$

$$
Y_{l, \mu-m_{e}}(\hat{q}) \mathcal{R}_{l e, l, \lambda, m_{\lambda}}^{i} \mathcal{A}_{l_{e}, m_{e}, l}^{\lambda, \mu}
$$




\section{RESULTS}

We first consider the simplest case of atomic hydrogen and compute the triply differential ionisation cross sections in the same conditions as the ones used in the work of Sahlaoui and Bouamoud [6] $E_{i}=250 \mathrm{eV}, E_{e}=5 \mathrm{eV}, \theta_{f}=5$ degrees.

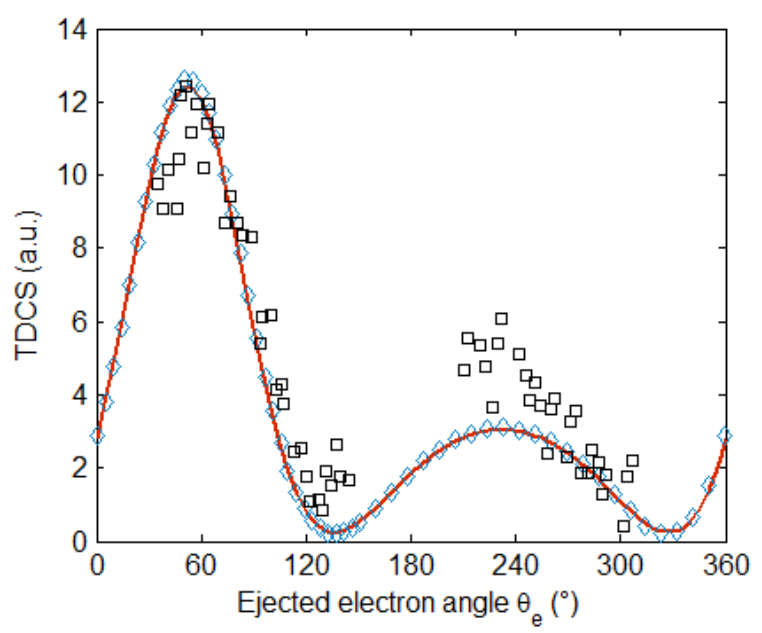

Fig. 1: Triply differential ionization cross sections of the ionization of atomic hydrogen. The considered kinematical conditions are: $E_{i}=$ $250 \mathrm{eV}, E_{e}=5 \mathrm{eV}$ and $\theta_{f}=5$ degrees. Black squares: experimental data [11], blue triangles: analytical data of Sahlaoui and Bouamoud [6], red solid line: our results.

Figure 1 shows that we numerically obtain exactly what was analytically found by Sahlaoui and Bouamoud with the same FBA-1CW model. The binary and recoil peak positions as well as the peak widths are in good agreement with the experimental data of Ehrhardt et al. [11] but the relative amplitude of the peaks is not well predicted by the FBA since the recoil peak's amplitude is underestimated.

The same shape of the TDCS as function of the ejected electron angle is found in the ammonia case under the kinematical conditions considered in the work of El Mir et al. [13]. In figure 2, the experimental data of El Mir et al. are represented by the solid black squares, their $1 \mathrm{CW}$ analytical results are given by the blue diamonds. Our numerical results are represented by the dashed green line in figure 2 for molecular wave functions calculated using Gaussian03 and by the red solid line for molecular wave functions calculated using the approach proposed by Moccia.

Good agreement is found between the analytical triply differential cross sections of El Mir et al. and our data calculated using Slater-type wave functions futher validating our program. The amplitude of the binary peak is a little different with the 6-31 Gaussian basis set than using the Moccia approach to develop the molecular wave function but in general the TDCSs have the same shape using both Slater and Gaussian type wave functions. In comparision with the experimental data, the width of the binary peaks and the amplitude of the recoil peaks are underestimated. This proves the need for a more detailed description of the interactions taking place in order to have a better estimation of the experimental data.

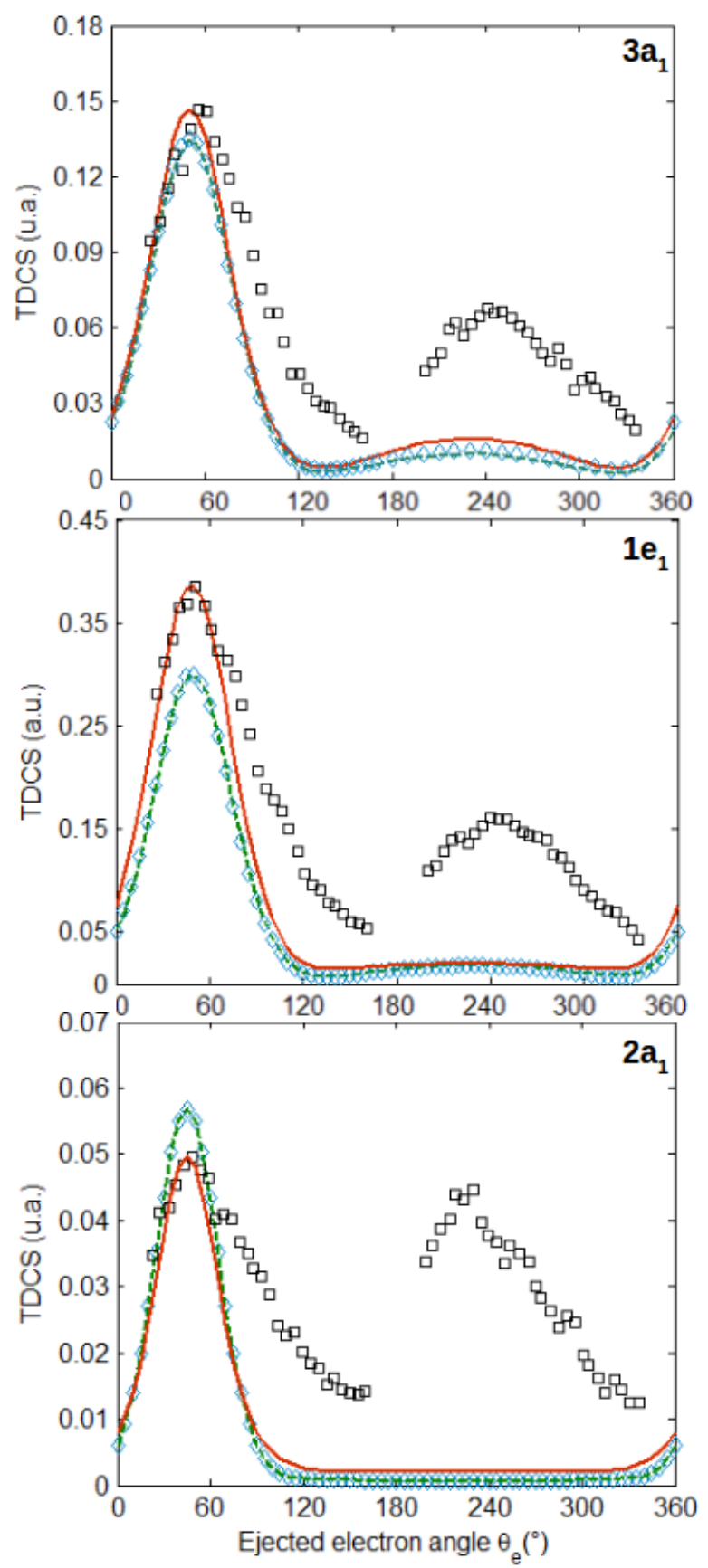

Fig. 2: Triply differential ionization cross sections of the ionization of the three outer valence orbitals of ammonia. The considered kinematical conditions are: $E_{i}=500 \mathrm{eV}, E_{e}=74 \mathrm{eV}$ and $\theta_{f}=-6$ degrees. Black squares: experimental data [13], blue triangles: analytical FBA-1CW data given in the same work, green dashed line: our results using Slater-type molecular wave functions, red solid line: our results using Gaussian-type molecular wave functions. 


\section{CONCLUSION}

We present a theoretical model using partial wave expansion and based on the First Born approximation to calculate the triply differential single electron impact ionization cross sections of hydrogen and ammonia. Partial wave expansion is an important tool that facilitates programming the theoretical model and using a computing grid to reduce computing time which is of particular interest for the study of big molecules. Using Gaussian 03 to develop single-center molecular wave functions makes the corresponding program user-friendly with the ability to easily change the parameters for different molecules and kinematical conditions. As a start, we demonstrate the validity of our program by comparing the results with previously published data for atomic hydrogen and present the triply differential cross sections for the ionization of ammonia based on this formalism which are in good agreement with published data. The same discrepancies between experimental and theoretical FBA data that were noted in a previous study were observed in our work in the case of the ammonia, especially regarding the inability of FBA$1 \mathrm{CW}$ model to accurately describe the collision process under the considered kinematics when an electron is ejected in the backwards direction. This work is a first step to validate the functions used in our program as well as the transition from Gaussian03 output to the program where the molecular wave function is also calculated. The next step would be to use the program to determine cross sections for more complex molecules such as the DNA bases and develop the collision description into a more complex description of the interactions between the projectile and the target.

\section{ACKNOWLEDGMENT}

This work is co-funded by IN2P3-CNRS and IRD, France under the ARTS research grant program. It is also supported by the Projet International de Coopération Scientifique (Pics 6509) between the French CNRS and the Lebanese CNRS, Lebanese University and Saint Joseph University.

\section{REFERENCES}

[1] C.-Y. Lin, C. McCurdy, and T. Rescigno, "Complex kohn approach to molecular ionization by high-energy electrons: Application to h 2 o," Physical Review A, vol. 89, no. 1, p. 012703, 2014.

[2] C. Champion, "Electron impact ionization of liquid and gaseous water: a single-center partial-wave approach," Physics in medicine and biology, vol. 55, no. 1, p. 11, 2009.

[3] I. Tóth, R. I. Campeanu, and L. Nagy, "Ionization of nh3 and ch4 by electron impact," The European Physical Journal D, vol. 69, no. 1, p. 2, 2015.

[4] K. Kaufmann and W. Baumeister, "Single-centre expansion of gaussian basis functions and the angular decomposition of their overlap integrals," Journal of Physics B: Atomic, Molecular and Optical Physics, vol. 22, no. 1 , p. $1,1989$.

[5] S. Houamer, M. Chinoune, and C. Dal Cappello, "Theoretical study of (e, 2e) process of atomic and molecular targets," The European Physical Journal D, vol. 71, no. 1, p. 17, 2017.
[6] M. Sahlaoui and M. Bouamoud, "Electron impact single ionization of the water molecule in the second born approximation," Journal of Physics B: Atomic, Molecular and Optical Physics, vol. 45, no. 8, p. 085201 , 2012.

[7] M. Sahlaoui and M. Bouamoud, "Cross sections for electron-impact ionization of water molecules," Canadian Journal of Physics, vol. 89, no. 6, pp. 723-727, 2011.

[8] J. Gao, J. Peacher, and D. H. Madison, "An elementary method for calculating orientation-averaged fully differential electron-impact ionization cross sections for molecules," The Journal of chemical physics, vol. 123, no. 20, p. 204302, 2005.

[9] E. Ali, K. Nixon, A. Murray, C. Ning, J. Colgan, and D. Madison, "Comparison of experimental and theoretical electron-impact-ionization triple-differential cross sections for ethane," Phys. Rev. A, vol. 92 , p. 042711, Oct 2015.

[10] M. Frisch, G. Trucks, H. Schlegel, G. Scuseria, M. Robb, J. Cheeseman, G. Scalmani, V. Barone, B. Mennucci, G. Petersson, et al., "Gaussian 09, revision b. 01. wallingford, ct: Gaussian,” Inc.,(2, 2004.

[11] H. Ehrhardt, K. Jung, G. Knoth, and P. Schlemmer, "Differential cross sections of direct single electron impact ionization," Zeitschrift für Physik D Atoms, Molecules and Clusters, vol. 1, no. 1, pp. 3-32, 1986.

[12] A. S. Kheifets, A. Naja, E. M. S. Casagrande, and A. Lahmam-Bennani, "Dwba-g calculations of electron impact ionization of noble gas atoms," Journal of Physics B: Atomic, Molecular and Optical Physics, vol. 41, no. 14, p. 145201, 2008.

[13] R. El Mir, E. S. Casagrande, A. Naja, C. Dal Cappello, S. Houamer, and F. El Omar, "Triple differential cross sections for the ionization of the valence states of nh3 by electron impact," Journal of Physics B: Atomic, Molecular and Optical Physics, vol. 48, no. 17, p. 175202, 2015.

[14] R. Moccia, "One-center basis set scf mo's. ii. nh3, nh4+, ph3, ph4+," The Journal of Chemical Physics, vol. 40, no. 8, pp. 2176-2185, 1964

[15] A. Lahmam-Bennani, A. Naja, E. S. Casagrande, N. Okumus, C. Dal Cappello, I. Charpentier, and S. Houamer, "Dynamics of electron impact ionization of the outer and inner valence (1t2 and 2a1) molecular orbitals of ch4 at intermediate and large ion recoil momentum," Journal of Physics B: Atomic, Molecular and Optical Physics, vol. 42, no. 16, p. 165201, 2009.

[16] C. Champion, C. Dal Cappello, S. Houamer, and A. Mansouri, "Single ionization of the water molecule by electron impact: Angular distributions at low incident energy," Physical Review A, vol. 73, no. 1, p. $012717,2006$.

[17] H. D. Hafied, Etude théorique de l'ionisation par impact électronique des molécules d'eau en phase gazeuse et liquide. $\mathrm{PhD}$ thesis, Metz, 2007. 